\title{
SHANDAWEEL 1: A NEW BREAD WHEAT(Triticum aestivum L.) CULTIVAR
}

Sadek, Eman M.; A.M.Tammam; M.B. Tawfelis; I. A. Amin; A. Hamada; A. Abo- Warda; S. Abdel- Majeed; M. Mesherf; E.A.M.EISayed; H. Ashoush; H. Hendawy;Hayam S. Mahgoub; A.K. Mostafa; H. El-Borhamy; A. Menshawy; A.Moussa;Wafaa M. ElAwady; Nadia Abd El-Nour; Sabah. Abo El-Ela; R. Kumber; A. Swelam; S. Abdel- Dayem; G. El- Shaaray; S. Sleem; Sohair M. Hassan; S. Hamad; M.A. Khaled; S. El- Sawy; Magda A. Abdel Rahman; Manal A. Hassan; M. A. El-Maghraby; Aza M. Abdel-Al; A. A. morad;R.A. Ramadan; M. Zakaria; A. Hagras; A.T. Mostafa; M.S. Mahmoud; M.Y.Moubark;ThanaaAbd-El-Kreem ;A.M.MorsyHodaEI-Gharabawy;Aglan,M.A.A; W. Farahat; Abd ElHamid E.A.M;K.Ragab;K.I.Gad;E; Nathan Shereen; I.S.M. AbdelLatif,; M.N. Abdel Kader; M.M.Mohiy;Gehan, A.N.; A. Gomaa; T. Shehab El Din; Mostafa, M. A.; M. Abo Shereef; S.R.Sabry; M.S. Sharshar; M. Abde-Aleem;M.A.Gouda;M.El-Menofy; A.Ageez; EnayatGhanem; S.Kh. Mahmoud; M.G.Mosaad; N.Hanna; M.A. Moussa; M.A. Salem; A. Khattab; A. Abdel-Latif;A. El-Hag; Najwa Abdel- Fattah; F. Hefnawy ; A. Gad-Allah; I. A. Imbaby*.and Doaa ,RaghebElnagar*

Wheat Res. Dept. Field Crops Res. Inst., ARC, Giza 12619, Egypt.

*Wheat Disease Dept., Plant Pathology Res. Inst., ARC, Giza 12619, Egypt.

\section{ABSTRACT}

The new promising bread wheat cultivar Shandaweel 1 has been selected from one of the advanced lines among the International Maize and Wheat Improvement Center collections ( CIMMYT )grown in wheat research department, Field Crops Research Institute, ARC. Egyptat Shandaweel Research Station. The grain yield of the new cultivar Shandaweel 1 was evaluated through 99 experiments conducted at four different levels; ( 8 macro, 56 micro and 35 verification yield trials)in 2005/2006, 2006/2007, 2007/2008and 2011/2012,seasons, respectively. Results proved superiority of the new bread wheat cultivar Shandaweel 1 compared to the commercial checks cultivars Sakha 93, Sakha 94, Gemmeiza 9 and Giza 168 for grain yield at North, South and Middle Delta and Upper Egypt regions. Moreover, the results of the verification yield trials ensure the superiority of Shandaweel 1 cultivar on the commercial wheat cultivar Giza 168 at North, Middle, South Delta and Upper Egypt. Alsothe superiority of Shandaweel 1 cultivar on the commercial wheat cultivar Giza 168 and Gemmeiza 9 at North and South Delta and the Out Valley region was observed and recorded.

Thus, we can highly recommended the new cultivar Shandaweel 1 to be grow at North Delta, South Delta,Middle Delta, Middle and Upper Egypt and the Out Valley regions. 


\section{INTRODUCTION}

Wheat is the most important cereal crop in terms of its total acreage and production; also it is a staple diet for more than one third of the world population. Wheat contributes more calories and protein to the world diet than any other cereal crop.

Increasing production / unit area appears to be the mainly possible alternative of reducing the wheat production gap. The required yield increases may be achieved by introducing high yielding cultivars and simultaneously implementing improved cultural practices. Such improved varieties must resist or tolerate the unfavorable environments and be stable in broad spectrum of environments.

Heat stress is a common abiotic stress that causes stunted plants, reduced tillering, and accelerates development leading to small heads, shriveled grains and finally translated to low yields(Tawfelis,2006). Respecting agronomic traits affected by this abiotic stress such as days to heading, days to maturity, plant height and grain yield can be found easily identifiable traits as indices for heat tolerance. Therefore, evaluation of breeding materials under different environments has to be done. Understanding the nature of genotype $x$ environment interaction empowers breeders to test and select of more efficient genotypes. Breeding genotypes with wide adaptability has long been a universal goal among plant breeders. To achieve this goal, evaluating breeding lines over time and space has becomes an integral part of any plant breeding program. Adaptability and stability performance of cultivars over environments are important for national policy in crop production, therefore a grain producer is interested primarily in growing a cultivar with high yield and stability performance at his location.

In mid $1990 \mathrm{~s}$, Sids 1, the commercial bread wheat cultivar was released for cultivation in Middle and Upper Egypt as well as in saline soils (Ghanem,Enayat et.al., 1996). Two high yielding bread wheat cultivars, Sakha 93 and Giza 168 were released by Shehab El-Din et.al.,in 1999 year, when they proved their superiority and wide adaptability to all Egyptian districts. Sakha 93 cultivar showed good tolerance to unfavorable environmental conditions, e.g. salinity and drought. In different successive series, further bread wheat cultivars, i.e. Gemmeiza 7 (Shehab El-Din et.al.,2000), Gemmeiza 9 (Mosaad et.al. 2000). Sakha 94 (Shehab El-Din et.al.,2005) , Gemmeiza 10 (El-Shami et. al.,2005) and Sids 12 (Mahrous et.al., 2009) were released during the last eight years, for the same goal.

The present investigation is actually an evaluation for yielding ability of the new developed bread wheat cultivar, Shandaweel 1, comparing to the superior Egyptian commercial cultivars. 


\section{MATERIALS AND METHODS}

The new promising cultivar Shandaweel1, has been selected from the exotic materials tested at Shandaweel Agricultural Research Station, Egypt. The cross and pedigree of the new cultivar is :

\section{Site / Mo / 4/ Nac / Th. Ac //3* Pvn /3/ Mirlo / Buc}

CMSS93 B00S 67S -72Y - 010M - 010Y - 010M - 3Y - OM - OTHY - OSH

To evaluate the yielding ability and rust resistance of the new cultivar Shandaweel 1, the following characters were studied:

1-Yield evaluation :

The new released cultivar Shandaweel 1, was tested in 2005/2006 growing season for grain yield ability against those of the commercial cultivars Sakha 93, Sakha 94, Gemmeiza 9 and Giza 168 in preliminary yield trials conducted at eight research stations, representing different locations, Sakha (North Delta), El-Gemmeiza (Middle Delta), Kafr-El Hamam (East Delta), Sids (Middle Egypt), Shandaweel, El-Matanaa and Kom- Ombo (Upper Egypt) and El- Nobariaa (Out Valley). The statistical design used in these trials was the randomized complete block design (RCBD) with four replicates according to Steel and Torrie (1980). The plot area was 4.2 $\mathrm{m}^{2}$ included 6 rows, $3.5 \mathrm{~m}$ long and $20 \mathrm{~cm}$ apart.

Moreover, Shandaweel 1, was tested in all over the country for yielding ability versus the same commercial cultivars (checks) in 28 advanced yield trials including 16 entries in 2006/2007 growing season and 27advanced yield trials in 2007/2008 growing season including 12 entries. In these advanced yield trials, all promising lines as well as the commercial wheat cultivars were tested in large plot area experiments ( $3 \times 3.5=10.5 \mathrm{~m}^{2}$ each). All recommended cultural practices for each region were applied on all trials.

For the demonstration aspects, 35 verification yield trials for Shandaweel 1, and the local checks were conducted in both old and new lands in 2011/2012 growing season. The area of each selected field was 500 $\mathrm{m}^{2}$ ( $100 \mathrm{~m}^{2}$ for each cultivar ). At harvesting time, five randomly selected samples (4 $\mathrm{m}^{2}$ each) from each plot were harvested and threshed. The kernels of each sample were weighted and adjusted to ardab/ faddanto estimate the grain yield.

\section{2-Disease severity;}

Disease severity score, expressed as the \% coverage of leaves with rust pustules and plant reaction, to indicate the infection type; $0=i m m u n e$ , $R=$ resistant, $M R=$ moderately resistant,MS=moderately susceptible and $S=$ Susceptible were recorded. Moreover, Average Coefficient of infection (ACl) was calculated using adapted scale of Saari and Wilcoxson (1974) as follows:

$\mathrm{O}=0.0, \mathrm{R}=0.2, \mathrm{MR}=0.4, \mathrm{X}($ mixed reaction $)=0.6, \mathrm{MS}=0.8$ and $\mathrm{S}=1.0$ 


\section{RESULTS AND DISCUSSION}

\section{1- Preliminary yield trials:}

The results in Table 1 shows the grain yields of the new cultivar Shandaweel 1 and the four bread wheat commercial cultivars, Sakha 93, Sakha 94, Gemmeiza 9 and Giza 168 in the preliminary yield trials conducted at eight research stations in 2005/2006 growing season. These research stations represent the old and new lands of Egypt. It is obvious from this table thatShandaweel 1 had significantly surpassed Sakha 93 at four out of the eight tested locations. Moreover, Shandaweel 1 was not significantly differed from the other three checks. The results indicated that Shandaweel 1 cultivar surpassed Sakha 93 cultivar in the preliminary yield trials by $13.63 \%$ over eight locations. The results alsoshow that Shandaweel 1cultivar exceeded Sakha 93 cultivar at El-Gemmeiza location by $12.20 \%, 39.68 \%$ at Kafr-El Hamam, $17.66 \%$ at Shandaweel and by $18.25 \%$ at El- Nobaria location.In addation,Shandaweel 1 cultivar produced thehighest grain yield at the over all mean locations compared withSakha 93, Gemmeiza 9 and Giza 168 cultivars.

Table 1: Grain yield (ard/fad) of the preliminary yield trials for Shandaweel 1 and four bread wheat cultivars in 2005/2006 season.

\begin{tabular}{|c|c|c|c|c|c|c|c|c|c|}
\hline \multirow[b]{2}{*}{ Cultivars } & \multicolumn{8}{|c|}{ Locations } & \multirow[b]{2}{*}{ Mean } \\
\hline & 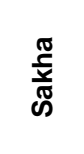 & 山 & 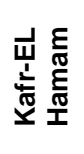 & $\frac{n}{\infty}$ & 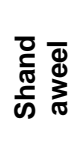 & 岀 & 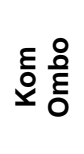 & 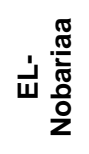 & \\
\hline Sakha 93 & 23.95 & 19.75 & 18.07 & 23.84 & 18.46 & 17.44 & 22.27 & 15.89 & 19.96 \\
\hline Sakha 94 & 24.85 & 20.17 & 24.35 & 22.75 & 23.61 & 16.05 & 23.92 & 17.75 & 21.67 \\
\hline Gemmeiza 9 & 23.36 & 21.66 & 23.80 & 22.86 & 20.85 & 14.85 & 20.76 & 20.96 & 21.14 \\
\hline Giza 168 & 22.88 & 21.74 & 21.96 & 23.30 & 22.37 & 18.68 & 22.67 & 17.68 & 21.41 \\
\hline Checks mean & 23.76 & 20.83 & 22.05 & 23.19 & 21.32 & 16.76 & 22.41 & 18.07 & 21.05 \\
\hline Shandaweel 1 & 25.13 & 22.16 & 25.24 & 24.94 & 21.72 & 19.04 & 21.41 & 18.79 & 22.30 \\
\hline LSD 5\% & 1.73 & 2.16 & 3.62 & 2.87 & 2.53 & 2.77 & 3.62 & 2.57 & 0.95 \\
\hline CV\% & 5.52 & 7.85 & 12.08 & 9.22 & 8.75 & 25.70 & 13.34 & 11.12 & 9.83 \\
\hline
\end{tabular}

\section{2-Advanced yield trials :}

The results of the advanced yield trials at North Delta in 2006/2007 season revealed that Shandaweel 1 significantly overcomethe four checks at one location only (Beheira) and had significantly exceeded the three checks, Sakha 93, Gemmeiza 9 and Giza 168at El-Serwand Sakha 93 and Giza 168 at Etai El-Barood location (Table 2-a). Meanwhile, the over all mean of North Delta, proved that,Shandaweel 1 mean was significantly higher than those of thethree checks, Sakha 93, Gemmeiza 9 and Giza 168while, the difference did not reach the level of significance withSakha 94. It could be stated thatShandaweel 1cultivarsignificantly surpassed Sakha 93, Gemmeiza 9 and Giza 168 cultivars by46.94, 51.12 and $27.92 \%$ at El-Serw location and 
exceeded the four checks, Sakha 93,Sakha 94,Gemmeiza 9 and Giza 168 by $12.30,25.94,49.03$ and $16.9 \%$, respectivelyatBehiraalocation. Meanwhile, the grain yield of the new bread wheat cultivar Shandaweel 1 exceededsignificantlythe two checksSakha 93 and Giza 168atEtai ElBarodandthe checkcultivar Gemmeiza 9at Sakha location. While, atTag ElEzz, Dakhlia 1 and Dakhlia 2 locationsnosignificantdifference between Shandaweel 1 and each ofthe four checks was detected .In addition, the results of the advanced yield trials atSouth Delta in 2006/2007 season shown in Table 2-b clearly indicated thatShandaweel 1cultivarsignificantly overcomethe four commercials wheat checks at one location only (Sharkia 2) and had significantly exceeded the three checks, Sakha 94, Gemmeiza 9 and Giza 168at Sers El-Liaan and Sakha 93, Sakha 94 and Giza 168at Kafr-El Hamam location. Meanwhile, in the over all mean of South Delta,Shandaweel 1cultivar significantly surpassed Sakha 93,Sakha 94,Gemmeiza 9 and Giza 168 cultivars by about 15.37, 11.58, 11.82 and $9.60 \%$, respectively.

Table 2a: Grain yield (ard/fad) of the advanced yield trials for Shandaweel 1 and four bread wheat cultivars at North Delta in $2006 / 2007$ season.

\begin{tabular}{|l|c|c|c|c|c|c|c|c|}
\hline \multirow{2}{*}{ Cultivars } & \multicolumn{7}{|c|}{ Locations } & \multirow{2}{*}{ Mean } \\
\cline { 2 - 9 } & EL-Serw & Sakha & $\begin{array}{c}\text { Tag EL- } \\
\text { EZZ }\end{array}$ & $\begin{array}{c}\text { Dakh } \\
\text { lia-1 }\end{array}$ & $\begin{array}{c}\text { Dakh } \\
\text { Lia2 }\end{array}$ & $\begin{array}{c}\text { Etai EL- } \\
\text { Barood }\end{array}$ & Beheira & \\
\hline Sakha 93 & 13.72 & 26.66 & 22.67 & 17.67 & 20.19 & 21.04 & 24.64 & 20.94 \\
\hline Sakha 94 & 16.36 & 27.05 & 21.40 & 17.00 & 20.36 & 24.87 & 21.97 & 21.29 \\
\hline Gemmeiza 9 & 13.34 & 25.58 & 19.40 & 12.00 & 20.96 & 24.54 & 18.57 & 19.20 \\
\hline Giza 168 & 15.76 & 27.43 & 19.27 & 14.67 & 17.35 & 23.90 & 23.67 & 20.29 \\
\hline Checks mean & $\mathbf{1 4 . 8 0}$ & $\mathbf{2 6 . 6 8}$ & $\mathbf{2 0 . 6 9}$ & $\mathbf{1 5 . 3 4}$ & $\mathbf{1 9 . 7 2}$ & $\mathbf{2 3 . 5 9}$ & $\mathbf{2 2 . 2 1}$ & $\mathbf{2 0 . 4 3}$ \\
\hline Shandaweel 1 & 20.16 & 28.42 & 20.40 & 13.74 & 17.45 & 26.37 & 27.67 & 22.03 \\
\hline LSD 5\% & 3.85 & 1.86 & 3.52 & 2.63 & 3.39 & 2.31 & 0.99 & 1.04 \\
\hline CV\% & 16.74 & 4.7 & 12.35 & 12.66 & 12.24 & 6.68 & 3.11 & 9.56 \\
\hline
\end{tabular}

Table 2b: Grain yield (ard/fad) of the advanced yield trials for Shandaweel 1 and four bread wheat cultivars at South Delta in 2006/2007 season.

\begin{tabular}{|c|c|c|c|c|c|c|c|c|}
\hline \multirow[b]{2}{*}{ Cultivars } & \multicolumn{7}{|c|}{ Locations } & \multirow[b]{2}{*}{ Mean } \\
\hline & 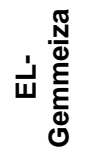 & 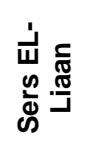 & 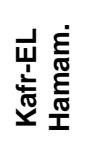 & $\frac{\bar{\pi}}{\frac{\pi}{\frac{1}{2}}}$ & $\begin{array}{l}\sim \\
\frac{\pi}{x} \\
\frac{ \pm}{\frac{\pi}{\pi}} \\
\frac{c}{\omega}\end{array}$ & $\begin{array}{l}\frac{\pi}{\frac{\pi}{5}} \\
\frac{\Sigma}{2} \\
\sum\end{array}$ & $\begin{array}{l}\frac{\pi}{0} \\
\frac{0}{2} \\
\frac{0}{0} \\
0\end{array}$ & \\
\hline Sakha 93 & 25.50 & 26.80 & 22.80 & 16.85 & 21.03 & 21.60 & 23.87 & 22.64 \\
\hline Sakha 94 & 27.04 & 21.47 & 22.01 & 19.92 & 24.17 & 26.07 & 23.17 & 23.41 \\
\hline Gemmeiza 9 & 24.94 & 20.04 & 25.48 & 19.86 & 18.46 & 26.60 & 28.14 & 23.36 \\
\hline Giza 168 & 27.30 & 22.84 & 22.81 & 17.70 & 23.65 & 27.94 & 26.40 & 24.09 \\
\hline Checks mean & 26.20 & 22.79 & 23.28 & 18.59 & 21.83 & 25.55 & 25.40 & 23.37 \\
\hline Shandaweel 1 & 28.40 & 27.84 & 26.06 & 18.84 & 27.00 & 27.60 & 27.07 & 26.12 \\
\hline \begin{tabular}{|l|} 
LSD 5\% \\
\end{tabular} & -- & 3.11 & 2.71 & 1.96 & 2.64 & 4.07 & -- & 1.22 \\
\hline CV\% & 5.09 & 8.79 & 8.16 & 7.47 & 8.09 & 11.06 & 14.60 & 9.67 \\
\hline
\end{tabular}


Table 2c: Grain yield (ard/fad) of the advanced yield trials for Shandaweel 1 and four bread wheat cultivars at Middle Egypt in 2006/2007 season.

\begin{tabular}{|l|c|c|c|c|c|c|}
\hline \multirow{2}{*}{ Cultivars } & \multicolumn{5}{c|}{ Locations } & \multirow{2}{*}{ Mean } \\
\cline { 2 - 6 } & Giza & Fayoum & Sids & Mallawy & Menia & \\
\hline Sakha 93 & 26.74 & 26.34 & 30.20 & 21.88 & 23.10 & 25.65 \\
\hline Sakha 94 & 29.75 & 27.00 & 28.67 & 19.50 & 21.35 & 25.25 \\
\hline Gemmeiza 9 & 26.88 & 27.34 & 28.57 & 19.66 & 23.10 & 25.11 \\
\hline Giza 168 & 29.19 & 28.47 & 28.64 & 19.58 & 24.50 & 26.08 \\
\hline Checks mean & $\mathbf{2 8 . 1 4}$ & $\mathbf{2 7 . 2 9}$ & $\mathbf{2 9 . 0 2}$ & $\mathbf{2 0 . 1 6}$ & $\mathbf{2 3 . 0 1}$ & $\mathbf{2 5 . 5 2}$ \\
\hline Shandaweel 1 & 28.57 & 26.34 & 29.50 & 20.27 & 20.30 & 25.00 \\
\hline LSD 5\% & 2.27 & -- & -- & 2.69 & 2.91 & 1.13 \\
\hline CV\% & 6.01 & 7.82 & 4.76 & 9.15 & 9.53 & 7.31 \\
\hline
\end{tabular}

Table 2d : Grain yield (ard/fad) of the advanced yield trials for Shandaweel 1

\begin{tabular}{|l|c|c|c|c|}
\hline \multirow{2}{*}{ Cultivars } & \multicolumn{3}{|c|}{ Locations } & \multirow{2}{*}{ Mean } \\
\cline { 2 - 4 } & Shandaweel & EL- Matanaa & KomOmbo & \\
\hline Sakha 93 & 20.84 & 23.47 & 14.65 & 19.65 \\
\hline Sakha 94 & 22.70 & 23.60 & 19.71 & 22.01 \\
\hline Gemmeiza 9 & 17.71 & 23.27 & 17.05 & 19.34 \\
\hline Giza 168 & 19.17 & 24.27 & 17.16 & 20.20 \\
\hline Checks mean & $\mathbf{2 0 . 1 1}$ & $\mathbf{2 3 . 6 6}$ & $\mathbf{1 7 . 1 5}$ & $\mathbf{2 0 . 3 0}$ \\
\hline Shandaweel 1 & 20.96 & 25.14 & 18.78 & 21.63 \\
\hline LSD 5\% & 2.02 & -- & 3.24 & 1.62 \\
\hline CV\% & 6.89 & 9.03 & 11.86 & 9.37 \\
\hline
\end{tabular}

Table 2 e : Grain yield (ard/fad) of the advanced yield trials for Shandaweel 1 and four bread wheat cultivars at Out Valley in 2006/2007 season.

\begin{tabular}{|c|c|c|c|c|c|c|c|}
\hline \multirow[b]{2}{*}{ Cultivars } & \multicolumn{6}{|c|}{ Locations } & \multirow[b]{2}{*}{ Mean } \\
\hline & Nobaria & Ismaliaa & Assuit & $\begin{array}{c}\text { New } \\
\text { Valley }\end{array}$ & Toshkey & Eoinaat & \\
\hline Sakha 93 & 26.65 & 7.27 & 14.87 & 14.67 & 17.32 & 15.07 & 16.00 \\
\hline Sakha 94 & 27.84 & 9.28 & 14.54 & 17.24 & 14.17 & 14.87 & 16.30 \\
\hline Gemmeiza 9 & 26.50 & 7.76 & 11.67 & 16.04 & 12.69 & 11.44 & 14.40 \\
\hline Giza 168 & 26.46 & 10.18 & 14.47 & 14.80 & 15.96 & 13.30 & 15.90 \\
\hline Checks mean & 26.86 & 8.63 & 13.88 & 15.69 & 15.04 & 13.67 & 15.65 \\
\hline Shandaweel 1 & 26.47 & 9.25 & 14.80 & 15.77 & 14.55 & 12.80 & 15.61 \\
\hline LSD 5\% & -- & 0.55 & 1.79 & 1.79 & 2.55 & -- & 0.90 \\
\hline CV\% & 8.16 & 3.79 & 8.64 & 8.00 & 12.14 & 14.19 & 9.95 \\
\hline
\end{tabular}

Concerning Middle Egypt region, data presented in Table 2-c showed that Shandaweel 1cultivar hadno significant difference in grain yield compared to the four wheatchecks either at the tested locations or in the over all mean of Middle Egypt region.

The results presented inTable 2-d for the advanced yield trials carried out at Upper Egypt in 2006/2007 season indicated that , the grain yield of the new bread wheat cultivar Shandaweel 1 exceeded Gemmeiza 9 cultivar by $18.35 \%$ at Shandaweel location andexceeded Sakha 93 cultivar by $30.78 \%$ at Kom-Ombo location. Also, The results showed thatShandaweel 
1cultivarsignificantly surpassed Sakha 93andGemmeiza 9cultivars atoverall locations

Furthermore, results of the advanced yield trials at Out Valley locations in 2006/2007 season indicated that the new bread wheat cultivar Shandaweel 1 showed insignificant difference in grain yield compared with the check cultivars at all locations and overall locations except producedsignificantly higher yield thanSakha 93 andGemmeiza 9 cultivars at Ismaliaa location andproduced greater yield compared to the cultivar Gemmeiza 9atToshkey ,Eoinaat andAssuit locations(Table 2-e).

The average grain yield of thewheat cultivar Shandaweel 1 compared to those of the commercial wheat cultivarsSakha 93, Gemmeiza 9 and Giza 168 in 27 advanced yield trials conducted at different sites all over the country in 2007/2008 growing season are shown in Tables ( $3 a, b, c, d$ and e).

Table 3a: Grain yield (ard/fad) of the advanced yield trials for Shandaweel 1 and three bread wheat cultivars at North Delta in 2007/2008 season.

\begin{tabular}{|c|c|c|c|c|c|c|c|}
\hline \multirow[b]{2}{*}{ Cultivars } & \multicolumn{6}{|c|}{ Locations } & \multirow[b]{2}{*}{ Mean } \\
\hline & EL-Serw & Dakhliaa1 & Dakhliaa2 & Sakha & $\begin{array}{l}\text { Etai EL- } \\
\text { Barod }\end{array}$ & Behiraa & \\
\hline Sakha 93 & 14.55 & 16.87 & 19.07 & 23.29 & 23.47 & 26.60 & 20.64 \\
\hline Gemmeiza 9 & 13.34 & 17.60 & 20.34 & 20.84 & 24.74 & 21.34 & 19.70 \\
\hline Giza 168 & 12.96 & 17.27 & 21.00 & 21.01 & 22.80 & 25.07 & 20.02 \\
\hline Checks mean & 13.62 & 17.25 & 20.14 & 21.71 & 23.67 & 24.34 & 20.12 \\
\hline Shandaweel 1 & 13.39 & 19.47 & 22.67 & 20.30 & 24.54 & 29.00 & 21.56 \\
\hline LSD 5\% & 1.80 & 1.56 & 1.35 & 2.18 & 1.59 & 1.95 & 0.70 \\
\hline CV\% & 9.51 & 6.32 & 4.74 & 6.99 & 4.62 & 5.57 & 6.11 \\
\hline
\end{tabular}

Table 3b: Grain yield (ard/fad) of the advanced yield trials for Shandaweel 1 and three bread wheat cultivars at South Delta in 2007/2008 season.

\begin{tabular}{|c|c|c|c|c|c|c|c|c|c|}
\hline \multirow[b]{2}{*}{ Cultivars } & \multicolumn{8}{|c|}{ Locations } & \multirow[b]{2}{*}{ Mean } \\
\hline & ய & 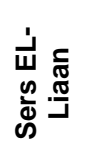 & 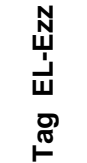 & 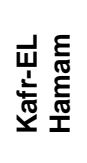 & $\begin{array}{l}\frac{\bar{\pi}}{\frac{1}{\frac{1}{\pi}}} \\
\frac{c}{\text { ⿹ }}\end{array}$ & $\begin{array}{l}\text { N } \\
\frac{\pi}{x} \\
\frac{1}{\frac{1}{\pi}} \\
\frac{c}{\omega}\end{array}$ & $\begin{array}{l}\frac{\pi}{5} \\
\frac{1}{2} \\
\sum\end{array}$ & $\begin{array}{l}\frac{\pi}{0} \\
\frac{0}{2} \\
\frac{0}{0} \\
0\end{array}$ & \\
\hline Sakha 93 & 24.58 & 26.17 & 16.90 & 20.05 & 21.64 & 16.54 & 23.60 & 28.67 & 22.27 \\
\hline Gemmeiza 9 & 24.26 & 21.94 & 19.05 & 18.98 & 20.17 & 19.27 & 24.80 & 26.54 & 21.88 \\
\hline Giza 168 & 24.29 & 26.37 & 20.10 & 20.65 & 21.40 & 19.14 & 24.20 & 27.94 & 23.01 \\
\hline Checks mean & 24.38 & 24.83 & 18.68 & 19.89 & 21.07 & 18.32 & 24.2 & 27.72 & 22.39 \\
\hline Shandaweel 1 & 24.73 & 28.70 & 21.76 & 16.47 & 18.20 & 22.07 & 22.60 & 23.07 & 22.20 \\
\hline LSD 5\% & 1.45 & 4.04 & 1.52 & 1.82 & n.s & 2.29 & n.s & n.s & 1.06 \\
\hline CV\% & 4.04 & 10.49 & 5.73 & 6.92 & 8.11 & 8.51 & 14.41 & 9.73 & 9.51 \\
\hline
\end{tabular}


Table 3c: Grain yield (ard/fad) of the advanced yield trials for Shandaweel 1 and three bread wheat cultivars at Middle Egypt in 2007/2008 season.

\begin{tabular}{|l|c|c|c|c|c|c|}
\hline \multirow{2}{*}{ Cultivars } & \multicolumn{5}{c|}{ Locations } & \multirow{2}{*}{ Mean } \\
\cline { 2 - 6 } & Giza & Fayoum & Sids & Mallawy & Menia & \\
\hline Sakha 93 & 25.00 & 31.85 & 26.07 & 20.58 & 20.33 & 24.77 \\
\hline Gemmeiza 9 & 25.00 & 33.95 & 22.40 & 19.78 & 19.62 & 24.15 \\
\hline Giza 168 & 26.34 & 30.80 & 22.14 & 21.60 & 22.76 & 24.73 \\
\hline Checks mean & $\mathbf{2 5 . 4 5}$ & $\mathbf{3 2 . 2}$ & $\mathbf{2 3 . 5 3}$ & $\mathbf{2 0 . 6 5}$ & $\mathbf{2 0 . 9 0}$ & $\mathbf{2 4 . 5 5}$ \\
\hline Shandaweel 1 & 25.67 & 32.38 & 26.00 & 20.32 & 15.41 & 23.96 \\
\hline LSD 5\% & 2.07 & 4.40 & 1.43 & 2.37 & 1.30 & 1.12 \\
\hline CV\% & 5.61 & 9.50 & 3.87 & 7.22 & 4.39 & 7.03 \\
\hline
\end{tabular}

Table 3d : Grain yield (ard/fad) of the advanced yield trials for Shandaweel 1 and three bread wheat cultivars at Upper Egypt in 2007/2008 season.

\begin{tabular}{|l|c|c|c|c|}
\hline \multirow{2}{*}{ Cultivars } & \multicolumn{3}{c|}{ Locations } & \multirow{2}{*}{ Mean } \\
\cline { 2 - 4 } & Shandaweel & EL- Matanaa & KomOmbo & \\
\hline Sakha 93 & 17.19 & 22.74 & 20.66 & 20.20 \\
\hline Gemmeiza 9 & 17.50 & 19.94 & 15.83 & 17.76 \\
\hline Giza 168 & 17.57 & 23.67 & 18.68 & 19.97 \\
\hline Checks mean & $\mathbf{1 7 . 4 2}$ & $\mathbf{2 2 . 1 2}$ & $\mathbf{1 8 . 3 9}$ & $\mathbf{1 9 . 3 1}$ \\
\hline Shandaweel 1 & 18.57 & 26.74 & 21.75 & 22.35 \\
\hline LSD 5\% & 2.73 & 3.81 & 3.56 & 1.91 \\
\hline CV\% & 10.16 & 11.00 & 13.30 & 11.55 \\
\hline
\end{tabular}

Table 3e:Grain yield (ard/fad) of the advanced yield trials for Shandaweel 1 and three bread wheat cultivars at Out Valley in 2007/2008 season.

\begin{tabular}{|l|c|c|c|c|c|c|}
\hline \multirow{2}{*}{ Cultivars } & \multicolumn{5}{c|}{ Locations } & \multirow{2}{*}{ Mean } \\
\cline { 2 - 6 } & Ismaliaa & Nobaria & Assuit & $\begin{array}{c}\text { New } \\
\text { Valley }\end{array}$ & Toshkey & \\
\hline Sakha 93 & 10.98 & 21.67 & 11.44 & 20.00 & 7.30 & 14.28 \\
\hline Gemmeiza 9 & 9.94 & 17.87 & 9.40 & 18.20 & 6.57 & 12.40 \\
\hline Giza 168 & 19.14 & 23.67 & 12.40 & 13.94 & 8.25 & 15.48 \\
\hline Checks mean & $\mathbf{1 3 . 3 5}$ & $\mathbf{2 1 . 0 7}$ & $\mathbf{1 1 . 0 8}$ & $\mathbf{1 7 . 3 8}$ & $\mathbf{7 . 3 7 3}$ & $\mathbf{1 4 . 0 5}$ \\
\hline Shandaweel 1 & 17.84 & 18.27 & 13.47 & 18.67 & 7.24 & 15.10 \\
\hline LSD 5\% & 1.02 & 2.71 & 2.06 & 1.78 & 0.95 & 0.79 \\
\hline CV\% & 4.29 & 8.70 & 10.57 & 6.62 & 9.45 & 8.20 \\
\hline
\end{tabular}

Date in Table3-a proved that Shandaweel 1 significantly exceededthecommercial cultivarsSakha 93, Gemmeiza 9 and Giza 168at Dakhlia 1, Dakhlia 2 , Beheira locations and in the over all mean of North Delta.Shandaweel 1 cultivarsignificantly exceededGiza 168cultivar only at Etai El-Baroodlocation.However, at Sakha location the cultivar Sakha 93 significantly out yielded the new cultivar Shandaweel 1 .

The results of the advanced yield trials at South Delta presented in Table 3-bindicated thatShandaweel 1cultivar had significantly surpassed the local checks at Tag El-Ezz,and Sharkia 2 locations and it had significantly overcomeGemmeiza 9 at Sers El Liaan locationwhile it had no significant 
differences with the three checks at four locations, namely El-Gemmeiza, Sharkia 1, Monufia and Qaluobia.

Data of the advanced yield trials of the Middle Egypt in 2007/2008 growing season presented in Table 3-c showed thatthe grain yield of the new bread wheat cultivarsShandaweel 1 had significantly exceeded Gemmeiza 9 and Giza 168 cultivars by $16.07 \%$ and $17.43 \%$, respectively, at Sids location. On the other hand, Shandaweel 1 had no significant difference comparedwith the three checks at the other locations; Giza, Fayoum,Mallawy, Menia and at over all meanof Middle Egypt.Meanwhile, the new cultivar Shandaweel 1significantly yielded less than the commercial wheat cultivarat Menia location.

For advanced yield trials in Upper Egypt(Table 3-d), the results showed that the new bread wheat cultivar Shandaweel 1yielded the same as the check cultivars at Shandaweellocation, while surpassed and exceeded significantlySakha 93 andGemmeiza 9 atEl-Matanaa location and Gemmeiza 9 only at Kom-Ombo location. The results clearly showed that Shandaweel 1 cultivar exceeded Sakha 93 by $10.7 \%$, Gemmeiza 9 by $25.84 \%$ and Giza 168 by $11.82 \%$ over all locations.

The grain yield of theadvanced yield trials at Out Valley in 2007/2008 season are presented in Table 3-e. The results proved that Shandaweel 1 cultivar was significantly superior thansakha 93 andGemmeiza 9atlsmaliaaand it had significantly exceeded Gemmeiza 9 only at Assiut location and Giza 168 cultivar at New Valley.The new wheat cultivar Shandaweel 1 significantly exceeded the yield of both Sakha 93 and Gemmeiza 9 cultivars at all over locations mean, while yielded almost the same as Giza 168 cultivar at new Valley location.

3-Verification yield trials

The results presented inTable 4 show the average grain yield estimated for 350n- Farm trials forShandaweel 1and some other cultivars which were carried at 22 Governorates representing the old and new lands in 2011/2012 growing season.

The results proved that the yield potentiality of the new cultivar Shandaweel 1 was almost the same as the local checks; Giza 168 and Gemmeiza 9 at Norath Delta, South Delta, Middle and Upper Egypt and New Lands region.

The grain yield of the new released cultivar Shandaweel 1 exceeded the check cultivar Giza 168 and was less than thecheck cultivar Gemmeiza 9 at Middle Delta (Dakahlia and Gharbia locations).Meanwhile, thegrain yield of the new cultivar Shandaweel 1 was less than the commercial wheat cultivar Giza 168 and Gemmeiza 9 at East Delta region. 
Sadek, Eman M. et al.

Table 4 : Grain yield (ard / fed) of verification yield trials of the newly released cultivar Shandaweel 1 in 2011/2012 season.

\begin{tabular}{|c|c|c|c|c|c|}
\hline \multirow{2}{*}{ Zones } & \multirow{2}{*}{ Governorates } & \multirow{2}{*}{$\begin{array}{l}\text { No.of } \\
\text { trials }\end{array}$} & \multirow{2}{*}{ Shandaweel 1} & \multicolumn{2}{|c|}{ Checks } \\
\hline & & & & Giza 168 & Gemmiza 9 \\
\hline \multirow{3}{*}{ North Delta } & Beheira & 2 & 23.94 & 20.86 & 22.63 \\
\hline & Alexandria & 2 & 20.65 & 22.26 & 22.88 \\
\hline & Kafr El-Sheikh & 4 & 21.21 & 22.52 & 19.33 \\
\hline \multicolumn{2}{|l|}{ Mean } & -- & 21.90 & 21.88 & 21.61 \\
\hline \multirow{2}{*}{ Middle Delta } & Dakahlia & 2 & 16.80 & 21.68 & 23.80 \\
\hline & Gharbia & 2 & 30.00 & 22.23 & 30.00 \\
\hline \multicolumn{2}{|l|}{ Mean } & -- & 23.40 & 21.96 & 26.90 \\
\hline \multirow{2}{*}{ South Delta: } & Monufia & 3 & 23.75 & 24.12 & 20.81 \\
\hline & Qalyuobia & 2 & 25.90 & 24.97 & 28.51 \\
\hline \multicolumn{2}{|l|}{ Mean } & -- & 24.83 & 24.55 & 24.66 \\
\hline \multirow{2}{*}{ EastDelta } & Ismailia & 1 & 17.50 & 24.50 & 21.00 \\
\hline & Port Said & 1 & 18.76 & 17.89 & 22.93 \\
\hline \multicolumn{2}{|l|}{ Mean } & -- & 18.13 & 21.19 & 21.96 \\
\hline \multirow{7}{*}{$\begin{array}{l}\text { Middle } \\
\text { andUpper Egyp }\end{array}$} & Giza & 2 & 28.35 & 29.05 & 27.30 \\
\hline & Fayoum & 2 & 26.20 & 27.65 & -- \\
\hline & Bani-Sweif & 2 & 19.95 & 19.25 & -- \\
\hline & Menia & 1 & 20.12 & 21.41 & -- \\
\hline & Assiut & 1 & 23.80 & 24.36 & -- \\
\hline & Sohag & 1 & 21.00 & 22.40 & -- \\
\hline & Aswan & 1 & 17.50 & 16.80 & -- \\
\hline \multicolumn{2}{|l|}{ Mean } & -- & 22.42 & 22.95 & 27.30 \\
\hline \multirow{6}{*}{ Out Valley } & Suez & 1 & 17.64 & 17.64 & 17.92 \\
\hline & Damietta & 1 & 12.20 & 10.08 & 11.02 \\
\hline & Nobaria & 1 & 20.53 & 17.73 & 14.93 \\
\hline & Bani-Sweif & 1 & 15.11 & 18.77 & -- \\
\hline & Qena & 1 & 13.60 & 11.40 & -- \\
\hline & New Valley & 1 & 15.11 & 18.00 & -- \\
\hline \multicolumn{2}{|l|}{ Mean } & -- & 15.70 & 15.60 & 14.62 \\
\hline \multicolumn{2}{|l|}{ Zones mean } & 35 & 20.44 & 20.70 & 21.77 \\
\hline
\end{tabular}

\section{4- Some technological and quality characters:}

Some technological and quality characters which are presented in Table 5 were carried out by Field Crops Technology Research Department ,Food Technology Research Institute, ARC. The results showed that the cultivar Shandaweel 1 had the highest hectoliter weight $(83.8 \mathrm{~kg}$.) while the other five cultivars were lowest in hectoliter weight and ranged from 80.3 for cultivar Gemmeiza 11 to 82.7 for the cultivar Sakha 94. This parameter is important for millers as it is positively correlated with the extraction rate (flour recovery). The extraction rate \% for Shandaweel 1 was the highest than the other five cultivars. 
Table 5: Some technological and quality characters of the new bread wheat cultivar Shandaweel 1 and five commercial wheat cultivars.

\begin{tabular}{|l|c|c|c|c|c|c|}
\hline \multirow{2}{*}{ Cultivar } & \multirow{2}{*}{$\begin{array}{c}\text { Hectoliter } \\
\text { weight } \\
\text { Kg. }\end{array}$} & Protein \% & Ash\% & \multirow{2}{*}{$\begin{array}{c}\text { Extraction } \\
\text { rate\% }\end{array}$} & Wet & Dry \\
\hline Sakha 93 & 82.1 & 10.0 & 1.8 & 70.0 & 21.8 & 7.2 \\
\hline Sakha 94 & 82.7 & 11.5 & 1.5 & 69.0 & 26.9 & 8.5 \\
\hline Gemmeiza 9 & 81.0 & 12.6 & 1.7 & 68.5 & 30.8 & 10.3 \\
\hline Gemmezia 11 & 80.3 & 12.2 & 1.7 & 69.0 & 24.3 & 9.0 \\
\hline Giza 168 & 82.1 & 12.0 & 1.8 & 68.3 & 28.8 & 9.1 \\
\hline Checks mean & 81.6 & 11.7 & 1.7 & 68.9 & 26.5 & 8.8 \\
\hline Shandaweel 1 & 83.8 & 12.5 & 1.9 & 71.3 & 26.5 & 9.7 \\
\hline
\end{tabular}

Protein content is an important parameter for making different products of wheat flour. Protein content \% estimates of the new bread wheat cultivar Shandaweel 1 along with the check cultivars Gemmeiza 9 and Gemmeiza 11 show a high protein content $>12 \%$ which are suitable for making good quality bread.

Wet gluten percentage of the six bread wheat cultivars which are presented in Table 5 show that the gluten \% of Gemmeiza 9 was the highest $(30.8 \%)$ followed by Giza 168 (28.8\%), Sakha 94 (26.9\%), Shandaweel 1 $(26.5 \%)$ and Gemmeiza $11(24.3 \%)$ while the cultivar Sakha 93 had the lowest value being $(21.8 \%)$. Similar rank was detected for dry gluten percentages where Gemmeiza 9, Shandaweel 1, Giza 168 , andGemmeiza 11 show values of $10.3 \%, 9.7 \%, 9.1 \%$ and $9.0 \%$, respectively. While the two check cultivars Sakha 93 and Sakha 94 recorded the lowest values of dry gluten percentages $7.2 \%$ and $8.5 \%$ respectively.

\section{5- Rust Disease Reaction:}

Data in Table 6 show the average response of the bread wheat cultivar Shandaweel 1 and four commercial wheat cultivars to leaf and stem rust disease at Gemmeiza, Sakha, and Nubaria Res. Stations in 2007/2008 season. The data revealed that Shandaweel 1 was completey resistant to leaf rust at Gemmeiza and Sakha stations and moderate resistant at Nubaria station( 10MR), it was completely resistant to stem rust at the three stations. In the same time, the other cultivars showed different reactions to leaf and stem rust ranged from resistant to susceptible responses.

Table 6-a : Mean average of leaf and stem rust diseases severity at adult stage for the new bread wheat cultivar Shandaweel 1 and four commercial wheat cultivars at Gemmeiza, Sakha, and Nubaria Res. Stations in 2007/2008 season.

\begin{tabular}{|l|c|c|c|c|c|c|}
\hline \multirow{2}{*}{ Cultivar } & \multicolumn{3}{|c|}{ Leaf Rust } & \multicolumn{3}{c|}{ Stem Rust } \\
\cline { 2 - 7 } & Gemmeiza & Sakha & Nubaria & Gemmeiza & Sakha & Nubaria \\
\hline Sakha 93 & $80 \mathrm{~S}$ & $60 \mathrm{~S}$ & $50 \mathrm{~S}$ & 0 & 0 & $20 \mathrm{~S}$ \\
\hline Gemmeiza 9 & 0 & 0 & $5 \mathrm{MS} / \mathrm{S}$ & 0 & 0 & $10 \mathrm{~S}$ \\
\hline Giza 168 & 0 & 0 & $20 \mathrm{MR} / \mathrm{MS}$ & 0 & 0 & $10 \mathrm{~S}$ \\
\hline Gemmezia 11 & 0 & $20 \mathrm{MS}$ & $30 \mathrm{MS} / \mathrm{S}$ & 0 & 0 & 0 \\
\hline Shandaweel 1 & 0 & 0 & $10 \mathrm{MR}$ & 0 & 0 & 0 \\
\hline
\end{tabular}

O=immune $\quad \mathbf{R}=$ resistance

MS= moderately susceptible

$\mathrm{S}=$ susceptible 
Table 6-b : Mean of average coefficient of leaf and stem rust diseases infection at adult stage for the new bread wheat cultivar Shandaweel 1 and four commercial wheat cultivars at Gemmeiza, Sakha, and Nubaria Res. Stations in 2007/2008 season.

\begin{tabular}{|l|c|c|c|c|c|c|}
\hline \multirow{2}{*}{ Cultivar } & \multicolumn{3}{|c|}{ Leaf Rust } & \multicolumn{3}{c|}{ Stem Rust } \\
\cline { 2 - 7 } & Gemmeiza & Sakha & Nubaria & Gemmeiza & Sakha & Nubaria \\
\hline Sakha 93 & 80 & 60 & 50 & 0 & 0 & 20 \\
\hline Gemmeiza 9 & 0 & 0 & 4.5 & 0 & 0 & 10 \\
\hline Giza 168 & 0 & 0 & 12 & 0 & 0 & 10 \\
\hline Gemmezia 11 & 0 & 16.00 & 24 & 0 & 0 & 0 \\
\hline Shandaweel 1 & 0 & 0 & 4.00 & 0 & 0 & 0 \\
\hline
\end{tabular}

From the previous results, it could be concluded that the new released cultivar Shandaweel 1 can be grown at North and South Delta, Upper Egypt and Out Valley and can share the dominating wheat cultivars at Middle Egypt to induce wide genetic variation which ensure higher degree of yield stability all over the country.

\section{REFERENCES}

El-Shami, M.; T. Shehab El-Din; M. Mostafa; M. Abdel- Aleem; M.Mahrous.; A. Ageez.; A. Hamada.; A.Bassiouni; M Eid; A. Abdel-Ghani; M. Eskander; S. Sabry; M. Sharshar; ImanSadek; A. Abo Warda; M. Moussa; S.Abdel-Majeed; A.Tammam; Najwa, Abdel-Fattah; M. Mosherf; E. El-Sayed; H. Ashoush; M.B. Tawfelis; HayamMahjoub; A. Moustafa; H. Hendawy; F.Hefnawy; S. Ali; A. Abdel - Karim; A. Menshowy; H. El-Borhamy; M. Abdel-Fattah G.El-Shaarawy; S. ElSawi; R. Kumber; Sabah Abo Elela; Wafaa EL-Awady; I. Amin; A. Moussa; S. Abdel Dayem; M.Zakeria; S. Hamamad; A. Swailem; A. Gomaa; O. Khalil; KadriaHegazi; EnayatGhanem; R. Mitkees; M. ElMonofy; A.M. Mousa; A. Abdel-Latif; N. Hanaa; A. Khattab and M. ElShamy. (2005). Gemmeiza 10; A new Egyptian high yielding and rust resistant bread wheat cultivar. J. Agric. Sci., Mansoura, Univ., 30 (2): $743-754$.

Ghanem, Enayat H.; A.MAbdelShafi; N.S.Hanna; S.Kh. Mahmoud; S.R.Sabry; M.M. Abde-Aleem; H.M.Zaid; M.A. Moussa; S. a. AbdelMajeed and S.Sherif (1996).Grain yield and stability of the new bread wheat "Triticumaestivum L" cultivar Sids 1 in different agronomic zones of Egypt. Bull.Fac.Agric. Univ., Cairo 47: 565-575.

Mahrous, A.M.; M. Abdel Aleem,; T. Shehab El-Din; M. Moustafa; S. AbdelMajeed; S. Sabry; Iman M. Sadek; M. Sharshar; A. Hamada; A. Abo Warda; A.M. Mousa; A.Tammam;M. Mosherf; E. El-Sayed; H. Ashoush; M.B. Tawfelis; H. Hendawy; Hayam S. Mahjoub; A. Mostafa; H. El-Borhamy; A. Menshawy; A. Mousa; Wafaa M. EL-awady; A. ElHag; R. Koumbor; S. Seleem; R.A. Ramdan; Nadia A. Abdel-Nour; G. Sharawy; SohairM.Hassan; A.Sewelam; S. El-Sawy; S. Abdel - 
Dayem; A.El-Sebaey; Magda A.Abdel- Rahman; Sabah H. Abo Elela; M. Khaled; I. Amin; M.Zakeria; Manal A, Hassan; A. Gad-Alla; S.Hamamad; M. El-Maghraby; A.Moard; Aza, M. Abdel-All; A. Hagras; A.T.Moustafa; M. Mahmoud; M. Moubark; KadriaHegazi; A. Gomaa; O. Khalil; Enayat.Ghanem; R. Mitkees; M. El- Monofy; S. Kh. Mahmoud; N. Hanna; M. Eid; M. Mosaad; M.A.Gouda; A.Ageez; M. Abdel-Fattah; A. Khattab; A. Abdel-Latif; M.Eskander; Najwa Abdel-Fattah; F.Hefnawy; and W. Abdel-Samad(2009). Grain yield and stability of the new Egyption bread whead cultivar Sids 12. J. Agric. Sci. ,Mansoura Univ., 34(4): 3199 -3209.

Mosaad M.; M. El-Monofy; T. Shehab El-Din; R.Mitkees; M. Mahrous; A. Hamada; A.Ageez; A.Bassiouni; M. El-Shami; M. Abdel-Aleem; M.Eid; A.Abdel-Ghani, M.Eskander, N.Hanaa; S.Sabry, A. Abdel-Latif; M.Sharshar; ImanSadek; M. Mostafa; A. Abo-Warda; Y. Abdel-Gwad; A. Mousa; S. Abdel-Majeed; A. Tammam; Najwa Abdel-Fattah; M. Moshref; E. El-Sayed; H. AshoushM.B. Tawfelis ; HayamMahjoub; A. Moustafa, F. Hefnawy, H. Hendawy; S. Ali; A. Abdel-Karim; A. Khattab; M. Abdel-Fattah, A. Menshawy, H. El-Borhamy; A. Gomaa, F. ElSayed; O. Khalil, KadriaHegazi; A. Ali, EnayatGhanem; S. Mahmoud and M. Khalifa(2000). Gemmeiza9. A new Egyptian high yielding and rust resistant bread wheat cultivar for delta region. J. Agric. Sci., Mansoura Univ., 25 (12): 7407 - 7419.

Saari, E.E.and R.D. Wilcoxson(1974).Plant disease situation of high yielding durum wheat in Asia and Africa.Arr.Rev.Phyto.;2:47-68.

Shehab El-Din , T. ; R.A. Mitkees , M.M. El-Shami ; M.A. Gouda; M.M. AbdelAleem; A.M. Abdel-Ghani; N.S. Hanna; S.R.S. Sabry; A.H. Abdel-Latif; M.S.A. Sharshar; Iman M.M. Sadek; A.M.A. Abo-Warda; M.Kh.. Moshref; E.A.M. El-Sayed; Hayam S. Mahgoub; A.K. Mostafa; M.G. Mosaad; A.H. Bassiouni; M.M.A. El-Menoufi; S. Kh. Mahmoud; M.A. Mahrous; A.A. Ageez; M.A.M. Eid; M.H. Iskandar; M.A. Mostafa; A.A. Hamada; Y.G. Abdel-Gwad; A.M. Mousa; S.A. Abdel-Majeed; A.M. Tammam; Nagwa R Abdel-Fatah; H.Ashoush; F.A. Hefnawy; H. Hendawy; S. El-DinAli;M.B. Tawfelis; A.A. Abdel-Kreem; A.A. Khttab; A.A. Gomaa; O.H.S. khalil; KadriaHegazi; EnayatH.Ghanem; A.A. Ali; F.F. El-Sayed; IkhlasShafik and S. Abo-Naga. (1999). Sakha 93 and Giza 168: Two new high yielding and rust diseases resistant bread wheat cultivars. J. Agric. Sci. ,Mansoura Univ., 24 (5): 2157 - 2168.

Shehab El-Din T., M. El-Monofy , M.Mosaad ; R. Mitkees; M.Mahrous; A. Hamada; A.Ageez; A.Bassiouni; M. El-Shami: M Abdel-Aleem; M.Eid; A.Abdel-Ghani, M.Eskander , N.Hanaa; S.Sabry, A. Abdel-Latif; M.Sharshar; ImanSadek; M. Mostafa; A. Abo-Warda; Y. Abdel-Gwad; A. Mousa; S. Abdel-Majeed; A. Tammam; Najwa Abdel-Fattah; M. Moshref; E.El-Sayed; H. Ashoush; M.B. Tawfelis; HayamMahjoub; A. Moustafa, F.Hefnawy, H. Hendawy; S. Ali; A. Abdel-Karim; A. Khattab; M. Abdel-Fattah, A. Menshawy, H. El-Borhamy; A. Gomaa, F. ElSayed; O. Khalil, KadriaHegazi; A. Ali, EnayatKhanem ; S. Mahmoud 


\section{Sadek, Eman M. et al.}

and S. Sherif. 2000. Gemmeiza 7: A new Egyptian long spike wheat cultivar. J. Agric. Sci., Mansoura Univ., 25 (11): 6709 - 6720.

Shehab El-Din T.; M. El-Shami; A. Abdel-Latif; M. Mostafa; M. Abdel- Aleem; M. Sharshar; A. Menshawy; H. El-Borhamy; S. Hamamad; M..Mahrous.; A. Ageez.; A.Bassiouni; M Eid; A. Abdel-Ghani; M. Eskander; S. Sabry; ImanSadek; A. Hamada.; A. Abo- Warda; S. Abdel-Majeed; A.Tammam; Najwa, Abdel-Fattah; M. Mosherf; E. ElSayed; H. Ashoush; M.B. Tawfelis; HayamMahjoub; A. Moustafa; F.Hefnawy; H. Hendawy; S. Ali; A. Abdel - Karim; M. Abdel-Fattah; G.El-Shaarawy; S. El-Sawi; R. Kumber; Sabah Abo Elela; Wafaa ELAwady; I. Amin; A. Moussa; A.M.Mousa; S. Abdel Dayem; M. Zakeria; A. Swailem; A. Gomaa; O. Khalil; KadriaHegazi; Enayatkhanem; R. Mitkees; M. El- Monofy; N. Hanaa; A. Khattab and W. Youssef( 2005). An introduction to Sakha 94, the new bread wheat cultivar. J. Agric. Sci., Mansoura Univ., 30 (1): $91-101$.

Steel, R.G.D. and J.H. Torrie(1980).Principles and Procedures of Statistics.Mc Grow-Hill Book Co., Inc., New York.

Tawfelis, M.B. 2006. Stability parameters of some bread wheat genotypes (Triticumaestivum) in New and Old Lands Under Upper Egypt conditions. Egypt .J. Plant Breed., 10(1): 223-246. 
إيمان محمد صادق , أحمد محمد تمسام , موريس بديع توفيلس , إبراهيم عبدالهادى أمين

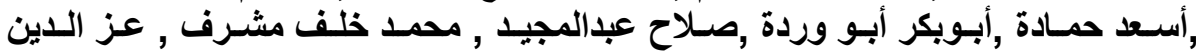

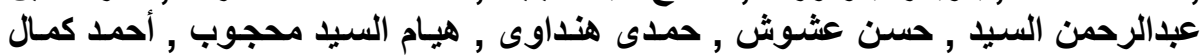

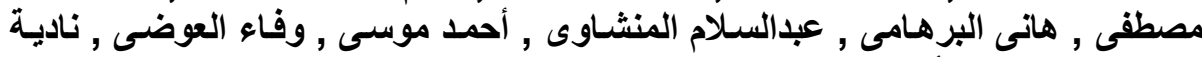

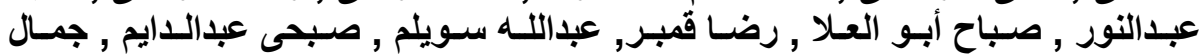

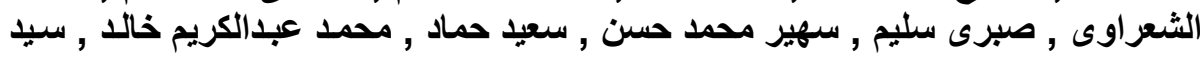

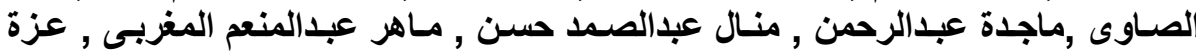

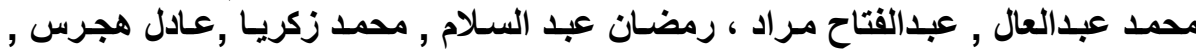

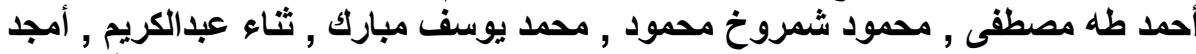

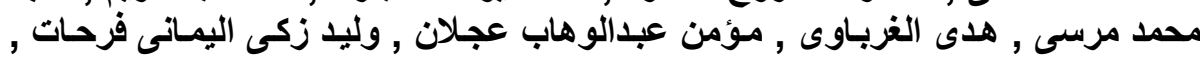

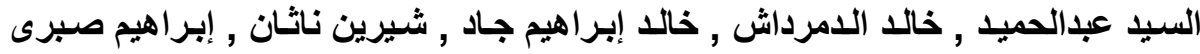

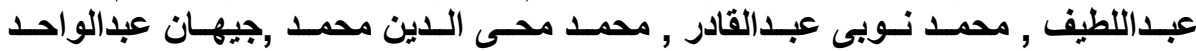

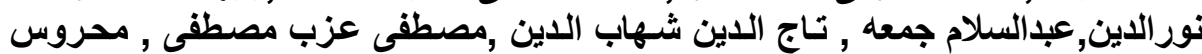

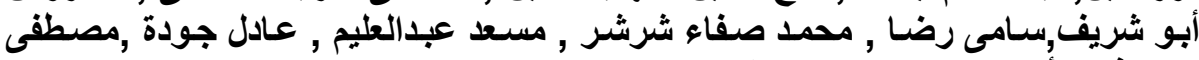

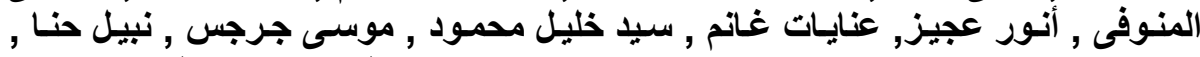

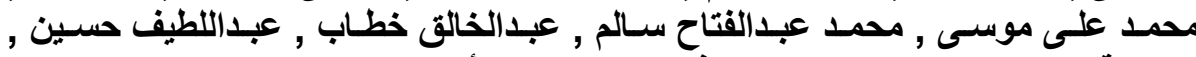

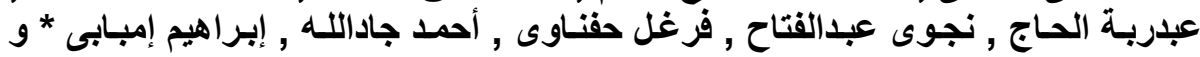

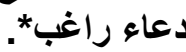

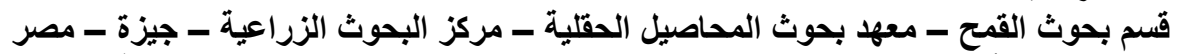

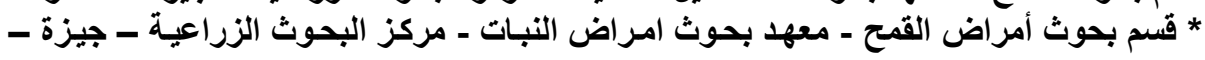

تم استتباط صنف جديد من قمح الخبز هو شندويل ا من قسم بحوث القمح فى محطة بحوث

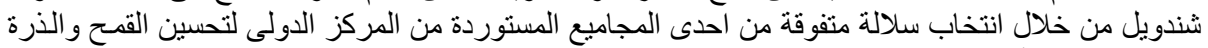

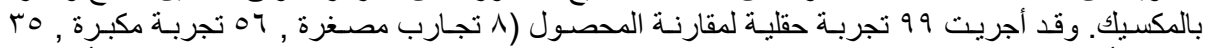

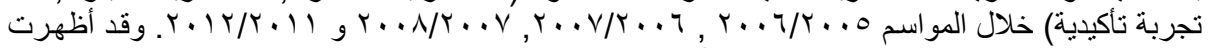

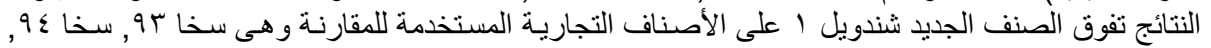

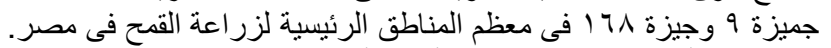

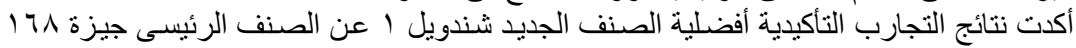

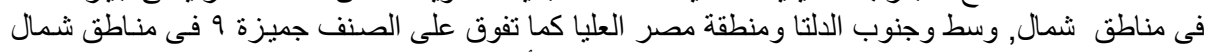

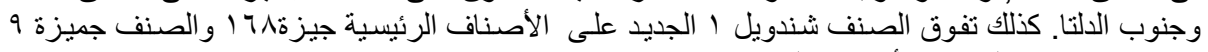
فى مناطق خارج الو ادى بالأراضى الجنديدة. ولهذا يوصى بزر اعة الصنف الجديد شندويل1 في مناطق شمال الدلتا, جنوب الدلتا، وسط الدلتا، مصر الوسطى , مصر العليا وفى الأراضى الجديدة.

كلية الزراعة - جامعة المنصورة كلية الزراعة - جامعة الزقازيق المنصوة
قام بتحكيم البحث

أ.د / أدمود سليمان اليحان سلطان أ.د / احمد عبد الغنى على لئل 